\title{
Thermal Management System for Press-Pack IGBT based on Liquid Metal Coolant
}

\author{
Yerasimos Yerasimou, Volker Pickert, Member, IEEE, Siyang Dai, Student Member, IEEE and \\ Zhiqiang Wang, Member, IEEE
}

\begin{abstract}
The press-pack packaging technology has been adopted in recent years for insulated-gate bipolar transistors (IGBTs) to be utilized in high voltage - high current applications, such as high-voltage direct current (HVDC) electric power transmission. Traditionally, the heat management of such systems is based on water coolant, however, there are numerous challenges associated with that method, such as the requirement to deionize the water to prevent electrical potentials and inherent problems of corrosion and leakage in the cooling piping structure. This paper presents the design and development of a liquid metal heat sink for press-pack IGBTs. The use of a thermal management system based on liquid metal increases the heat dissipation capability without corroding the cooling structure. Analytical work is performed on the design of the heat sink. Moreover, the thermal performance of the heat sink is experimentally validated against a commercial water-based cooling system. The presented results show that the cooling performance of the liquid metal system is increased, whereas the shortcomings of the water-based system are eliminated.
\end{abstract}

Index Terms-Press-pack insulated-gate bipolar transistor (IGBT) power module cooling, junction temperature, liquid metal cooling, magnetohydrodynamic (MHD) pump, high-voltage direct current (HVDC) cooling.

\section{INTRODUCTION}

$I^{2}$ NSULATED-gate bipolar transistors (IGBTs) are the most common power electronic devices for power conversion systems over $600 \mathrm{~V}$ [1]. The conventional packaging technology for IGBTs uses soldered bond wires for electrical connection and screw contacts for the power terminals. However, the long-term reliability of this packaging technology is compromised by its structure and materials. Due to the mismatch in the coefficient of thermal expansion between adjacent layers of different materials (see Fig. 1a), failures such as bond wire lift-off [2] and solder joint fatigue [3] occur as the result of thermomechanical stress experienced during temperature fluctuations.

Alternatively, for high current and voltage applications, press-pack IGBT (PPI) packaging technology, consisting of

This paragraph of the first footnote will contain the date on which you submitted your paper for review. It will also contain support information, including sponsor and financial support acknowledgment. For example, "This work was supported in part by the U.S. Department of Commerce under Grant BS123456."

The next few paragraphs should contain the authors' current affiliations, including current address and e-mail. For example, F. A. Author is with the

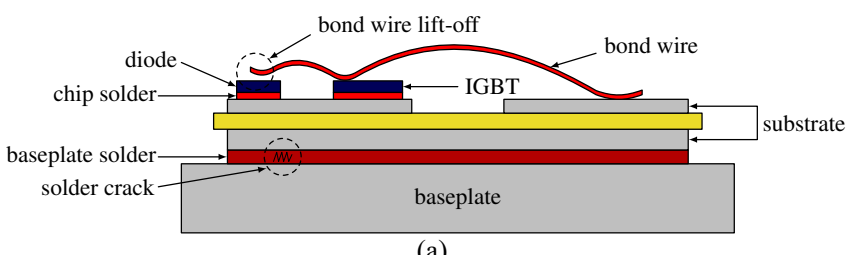

(a)

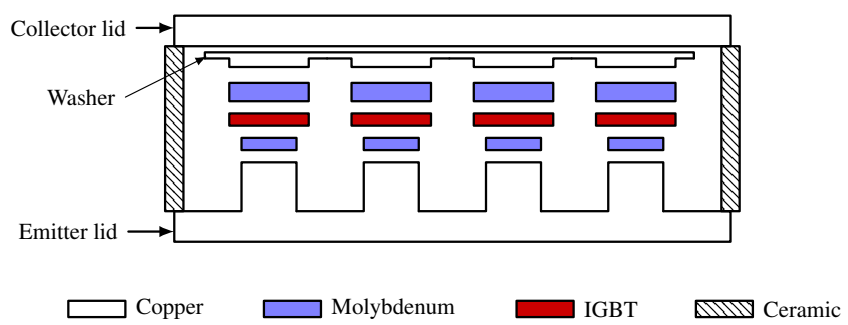

(b)

Fig. 1. Schematic of (a) conventional power module and (b) press-pack.

multiple individual IGBT chips arranged in parallel, has been developed to overcome the shortcomings associated to the conventional packaging technology. The PPI packaging technology is based on the packaging of monolithic devices that contain a single wafer, such as diodes, thyristors, and gate turnoff thyristors [4]. In contrast to the conventional power modules, where soldered bond wires are used for the electrical connections, press-pack devices achieve electrical and thermal contact by applying pressure, which is typically from 10 to 20 $\mathrm{N} / \mathrm{mm}^{2}$ [5]. Fig. 1(b) shows the schematic diagram of a typical PPI. Its structure consists of copper lids and molybdenum plates on each side of the silicon dies. Ceramic walls are used to hermetically seal the capsule. Molybdenum is utilized as a support material between the lid and the IGBT chip, as it has high mechanical hardness. In addition, it owns a coefficient of thermal expansion (CTE) similar to silicone's, therefore, any reliability issues associated with the mismatch of CTE between the materials of conventional power modules are significantly reduced. Combined with the absence of bond wires, the reliability of press-pack devices is significantly improved.

National Institute of Standards and Technology, Boulder, CO 80305 USA (email: author@boulder.nist.gov).

S. B. Author, Jr., was with Rice University, Houston, TX 77005 USA. He is now with the Department of Physics, Colorado State University, Fort Collins, CO 80523 USA (e-mail: author@lamar.colostate.edu).

T. C. Author is with the Electrical Engineering Department, University of Colorado, Boulder, CO 80309 USA, on leave from the National Research Institute for Metals, Tsukuba, Japan (e-mail: author@nrim.go.jp). 
Another advantage of press-pack package is the double-sided cooling, which allows for dissipating the excess heat more effectively. Moreover, unlike conventional IGBT power modules, which behave as an open circuit at their end of lifetime, PPIs feature a short-circuit failure mode. Finally, they are mechanically compatible to thyristor valves, and thus, they can be retrofitted to existing high-voltage systems or be used in new systems in conjunction with existing equipment. These features are particularly important for high-voltage direct current (HVDC) power applications, where multiple switching devices are connected in series to attain the required blocking voltage.

The heat management of press-pack devices in HVDC applications is challenging, as an effective cooling method that is able to cope with the high heat loads is required. The most common approach is to use water for dissipating the excess heat. However, since the current should also conduct through the heat sinks, deionised water should be used to avoid shortcircuiting the devices. Nonetheless, ionised particles are still developed within the coolant, which lead to corrosion of the piping system or blockage [6].

Liquid metals have gained attention in the past few years for electronics thermal management. They have excellent thermophysical properties, including very high thermal conductivity and low melting point. In addition, they own very high electrical conductivity; an important attribute for driving the fluid with an electromagnetic pump without any moving parts $[7,8]$. Comparisons between liquid metal and water for mini- and micro-channels show that liquid metal can achieve up to $40 \%$ reduction of the heat sink's thermal resistance [9]. Moreover, heat fluxes greater than $1500 \mathrm{~W} / \mathrm{cm}^{2}$ have been successfully dissipated [10]. Therefore, liquid metals are ideal for applications where high heat fluxes need to be dissipated and high reliability is required.

This work focuses on an alternative heat dissipation system for PPIs used in HVDC applications that can deliver high cooling performance and eliminate the disadvantages of using deionised water coolant. A thermal management system based on liquid metal coolant driven by integrated magnetohydrodynamic (MHD) pumps is proposed in this paper. The excess heat is transported to a liquid metal-to-water heat exchanger and finally dissipated to the ambient.

The rest of the paper is structured as follows. Section II presents the challenges for press-pack thermal management systems. The principle of operation for MHD pumps is presented in Section III. The design of the proposed heat sink for PPIs based on liquid metal is shown in Section IV. The experimental setup and results are presented in Section V. Finally, Section VI concludes the work.

\section{Current Technologies for Thermal Management OF PPIs}

Traditional cooling for HVDC PPI systems requires deionized water flowing through copper heat sinks that are configured in either typical "S" meander or "Archimedes" spiral structures [11]. The heat sink housing is made of copper due to its high electrical and thermal conductivities, which is nickel

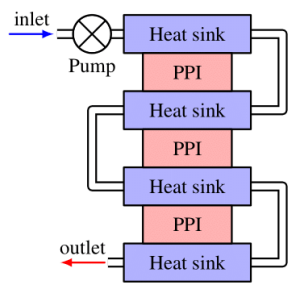

(a)

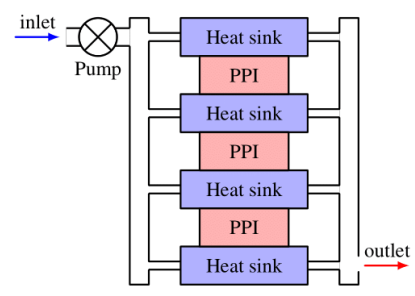

(b)
Fig. 2. Water-cooled PP stack configurations: (a) series and (b) parallel.

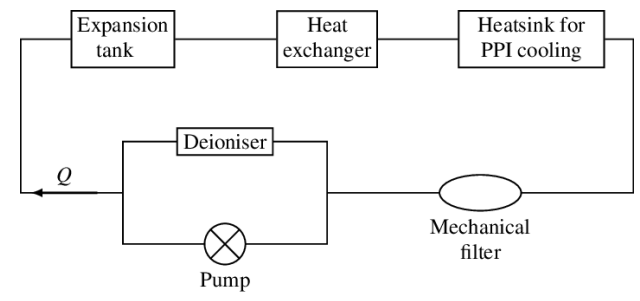

Fig. 3. Typical cooling system for HVDC using deionized water.

plated for protection against corrosion. The heat sinks are series or parallel connected and supplied through a liquid pump, as shown in Fig. 2.

Fig. 3 shows the schematic of a cooling circuit for HVDC, using deionized water as heat transfer medium. The water is circulated via a mechanical pump through the water heat sinks that cool the PPIs. The excess heat from the PPIs is dissipated in the heat exchanger. Deionized water also passes through an expansion tank that regulates the water pressure in case it rises due to temperature elevation. A mechanical filter collects any residuals in the water, whereas a deionizer is utilized to limit ionized particles that are developed in the water.

Deionized water is an electrolytic conductor; hence, its chemical composition varies as current conducts through it. Therefore, the free oxygen produced due to the electrolytic activity within the water contributes to the oxidization process of the cooling system [12]. In addition, any electrical potential imbalance between metal components of the cooling circuit, such as the heat sink body and pipe fittings, leads to chemical reactions on the metals. Therefore, in order to reduce the electrolytic currents flowing in the cooling medium, techniques such as limitation of the coolant's electrical conductivity via a chemical filter placed in a bypass parallel to the main circuit [13], minimization of the hydraulic diameter of the piping system [14] and placement of grading electrodes at predefined locations [15], should be employed. In addition, due to deionized water's aggressiveness irrespectively of whether current is flowing through it, the system should be pressurized so as the free oxygen content is limited. Even when plastic tubing, fittings or sealants are used, contact with deionized water could lead to embrittlement and thus, to premature failure [16]. Also, there is a risk of pit corrosion in areas where there is a contact of rubber sealants with metal surfaces [14]. In addition to the water based cooling, air cooled technologies are utilized for dissipating heat generated by press pack devices [17]. 
Nonetheless, their application is only limited to medium and low power ratings.

The main advantages of liquid metal cooling are the ability to drive the coolant by using a pump without moving parts and is therefore more reliable, and the utilization of a heat transfer medium that is more effective than water. In addition, due to the challenges mentioned earlier, there is need for a cooling method that is not prone to corrosion and premature failures. The main drawback of liquid metal coolants is the increased cost of the cooling mechanism, compared to traditional cooling mediums. A thermal management system based on liquid metal cooling agent is proposed in this work. Therefore, corrosion due to ionized particles is eliminated while the cooling capability of the system is maintained.

\section{MHD PUMP}

To overcome the limitations of the existing thermal management schemes a liquid metal based cooling system is presented in this work. The liquid metal is driven in a closed loop via an MHD pump that is integrated in the heat sink body.

\section{A. Governing equations of the MHD problem}

Magnetohydrodynamics is the branch of physics dealing with the flow of an electrically conductive fluid in the presence of a magnetic field. The term magnetohydrodynamics consists of the components magneto-, hydro-, and -dynamics that stand for the magnetic field, water or any other fluid medium and the motion of the object, respectively. The movement of the fluid can either be accomplished by external forces, in which case the movement of fluid in the presence of a magnetic field will generate an electric current, or by the interaction of the magnetic field and an imposed electric field, where a force is generated driving the fluid.

The physical laws governing the MHD phenomenon for a Newtonian fluid flow are a combination of electromagnetism and classical fluid dynamics that include Maxwell's equations, Ohm's law and Navier-Stokes conservation of mass and momentum, as well as energy equations. As this paper deals with the implementation of a liquid metal heat sink for presspack IGBT devices, the presented governing equations are only limited to Newtonian fluids.

The simplified Maxwell's equations that include magnetohydrodynamic components are:

$$
\begin{aligned}
& \nabla \cdot \boldsymbol{E}=\frac{\rho_{e}}{\varepsilon_{0}} \\
& \nabla \cdot \mathbf{B}=0 \\
& \nabla \times \boldsymbol{E}=-\frac{\partial \mathbf{B}}{\partial t} \\
& \nabla \times \mathbf{B}=\mu_{0} \mathbf{J}
\end{aligned}
$$

where $\boldsymbol{E}$ is the electric field, B is the magnetic field strength, $\mathbf{J}$ is the current charge density, $\rho_{e}$ is the charge density, $\varepsilon_{0}$ is the vacuum permittivity and $\mu_{0}$ the vacuum permeability. In these equations, it is assumed that the magnetic field is solenoidal and therefore, magnetic monopoles do not exist. In addition, magnetic fields could be generated by currents and electric fields could be generated by varying magnetic fields.
Moreover, according to Ohm's law, the current charge density $\mathbf{J}$ is proportional to the force experienced by free charged particles. For a conductive fluid with an electrical conductivity $\sigma$ moving with velocity $\mathbf{u}$ in a magnetic field $\mathbf{B}$, the current charge density $\mathbf{J}$ can be expressed as:

$$
\mathbf{J}=\sigma(\boldsymbol{E}+\mathbf{u} \times \mathbf{B})
$$

For liquid metal pump applications, the cross product $\mathbf{u} \times \mathbf{B}$ in (5) can be interpreted as an apparent electric field. Considering the case of electrical machines, $\mathbf{u} \times \mathbf{B}$ is analogous to the back EMF of the machine, whereas the fluid flow is analogous to the armature voltage. The power produced by the pump is maximized when $\mathbf{B}, \boldsymbol{E}$ and $\mathbf{u}$ are mutually orthogonal, in which case $\mathbf{u} \times \mathbf{B}$ is in the opposite direction of $\mathbf{J}$. Moreover, a Lorentz force is acting on a particle with a given charge that is moving with velocity $\mathbf{u}$, which is the sum of electrostatic Coulomb forces and magnetic forces. In MHD, the bulk forces acting on the medium are dominating the fluid flow, hence the force per unit volume acting on the conductor is:

$$
\mathbf{F}=\rho_{e} \boldsymbol{E}+\mathbf{J} \times \mathbf{B}
$$

As the fluid used in most cases has a very high electrical conductivity and its velocity is very small compared to the speed of light, the Coulomb force is negligible and can be ignored. Therefore, only the magnetic force is considered:

$$
\mathbf{F}=\mathbf{J} \times \mathbf{B}
$$

In addition to the electromagnetic equations, the MHD phenomenon also involves fluid dynamics equations to characterize the flow of the medium. The fundamental laws of fluid dynamics are based on Navier-Stokes conservation of mass, momentum and energy, and the governing equations describing the fluid flow can be written as:

$$
\begin{gathered}
\nabla \cdot \mathbf{u}=0 \\
\rho\left(\frac{\partial \boldsymbol{u}}{\partial t}+(\boldsymbol{u} \cdot \nabla) \boldsymbol{u}\right)=-\nabla p+\mu \nabla^{2} \boldsymbol{u}+\mathbf{J} \times \mathbf{B} \\
\rho C_{p}\left(\frac{\partial T}{\partial t}+(\boldsymbol{u} \cdot \nabla) T\right)=k \nabla^{2} T+\frac{\mathrm{J}^{2}}{\sigma}
\end{gathered}
$$

where $\rho$ is the fluid density, $p$ is the pressure, $\mu$ is the dynamic viscosity and $C_{p}$ is the specific heat capacity of the fluid. In (9), the momentum equation is modified for MHD applications by including the electromagnetic force component, $\mathbf{J} \times \mathbf{B}$. The term $\mathbf{J}^{2} / \sigma$ in (10) accounts for Joule heating caused by the injected current.

\section{B. MHD pump cooling applications}

The interaction of magnetic and electric fields is utilized in various applications for driving electrically conductive fluids. Compared to conventional pumps, they offer numerous advantages. The most important is the lack of moving parts. Because of that, the pump can provide silent operation, high reliability and minimal maintenance requirements. In addition, the pump can operate at very high temperatures and 
demonstrate high efficiencies. They have been successfully used for a range of applications, including seawater thrusters for ship propulsion [18-20], liquid metal stirring and shaping for industrial applications [21-23], molten salt pumping in nuclear reactors cooling [24, 25], blood pumping [26], sample propelling [27] etc.

In addition, a few instances have been reported where MHD pumps have been used for liquid metal cooling of electronic devices. The design and development of thermal management systems based on liquid metal for central processing units (CPUs) has been reported on multiple occasions in the past decade, demonstrating significant increase in the cooling performance compared to commercial heat sinks [28-31]. Liquid metal heat sinks have also been utilized for cooling high-power laser diodes [32] and adjusting their wavelength by controlling the coolant's flow rate [33]. Moreover, they have been used for cooling high-power light emitting diodes (LEDs) [34]. Liquid metals are also ideal for integrated cooling mechanisms for electronic devices in spatial restricted applications with local hotspots [35], as well as an alternative to heat pipes utilized as heat spreaders for orientation sensitive applications [36]. In addition, a liquid metal coolant has been proposed for reducing the weight of the power electronic cooling system for an aerospace application while maintaining similar cooling performance, in spite of its higher density [37]. Also, mini-channel cooling that can effectively dissipate 300 $\mathrm{W} / \mathrm{cm}^{2}$ [38] and ceramic mechanical pump, able to operate for temperatures up to $1400^{\circ} \mathrm{C}$, have been reported [39].

Experimental investigations of liquid metal mini-channel heat sinks aimed at power electronic applications have been reported demonstrating increased cooling performance over traditional cooling mediums [40, 41]. Moreover, an adaptive thermal management system based on liquid metal was successfully employed for reducing the thermal stress within the power module structure, and thus, increase its lifetime [42]. It must be noticed that all of the above listed work use an external current source to adjust $\mathbf{J}$ to get the MHD pump functioning.

\section{MHD pump principle of operation}

A schematic representation of the principle on which DC conduction pumps operate is shown in Fig. 4. The working fluid moves in a channel of width $a_{c h}$, height $b_{c h}$, and length $c_{c h}$ with the magnetic and electric fields placed perpendicular, so as to maximize the Lorentz force. The Lorentz force exerts body force $F$ on the fluid that can be expressed as:

$$
F=\int(\mathbf{J} \times \mathbf{B}) \cdot \mathrm{d} V \approx B_{\text {eff }} \cdot \mathrm{I}_{\mathrm{pump}} \cdot \mathrm{a}_{\mathrm{ch}}
$$

where $\mathrm{d} V$ is the active volume of the pump (i.e. the volume of the fluid under the presence of electric and magnetic fields), $B_{\text {eff }}$ is the efficient magnetic flux density, and $\mathrm{I}_{\text {pump }}$ is the current through the electrodes. As the channel width $b_{c h}$ is the distance between the electrodes, it is considered to be the conductor length (i.e. the current flow distance). The direction of the flow is dictated by the direction of electric and magnetic fields. Equation (11) assumes that $\mathbf{B}$ and $\mathbf{J}$ are homogeneous

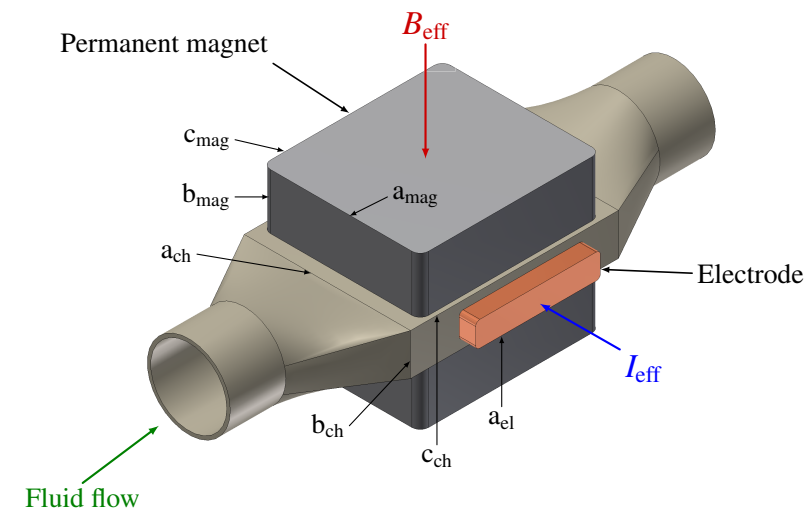

Fig. 4. Schematic of a typical MHD pump.

and constant. As shown in (12), the pressure developed by the pump is only a function of the magnetic field strength, the injected current, and the pump height $\mathrm{b}_{\mathrm{ch}}$ :

$$
P_{\mathrm{MHD}}=\frac{F}{A} \approx \frac{B_{\text {eff }} \mathrm{I}_{\mathrm{pump}}}{\mathrm{b}_{\mathrm{ch}}}
$$

where $P_{\mathrm{MHD}}$ is the pressure head and A is the cross section of the pump with width $a_{c h}$ and height $b_{c h}$. The flow rate can be calculated based on (13), where $C_{1}$ and $C_{2}$ are coefficients of flow resistance through the pump, considering the pump geometry and piping.

$$
Q=u A=\left(\left(C_{2}{ }^{2}+\frac{8 B_{\text {eff }} I_{\text {pump }} C_{1}}{\rho \pi r}\right)^{\frac{1}{2}}-C_{2}\right) \cdot \frac{\pi r^{2}}{2 C_{1}}
$$

\section{Design of Press-Pack Heat Sink}

The geometry of the heat sink with the integrated MHD pump was designed considering numerous parameters, such as the geometry of the PPI used for experimental validation, the magnetic field strength in the liquid metal pump active channels and the mechanical rigidity of the heat sink. A pre-assembled commercial PPI stack that consists of a pressure clamp and two PPI devices (Westcode T0360NB25A), which are double-sided cooled by water heat sinks, is used in this work. The schematic representation of the press-pack stack is illustrated in Fig. 5. Two pre-calibrated springs are used to ensure that constant pressure is delivered by the clamp during temperature variations that can potentially cause elastic deformation of the various components. Moreover, mechanical pressure indicators are placed on both sides of the clamping mechanism to ensure that sufficient clamping force is supplied to the press-pack stack.

The proposed liquid metal heat sink is designed based on the geometry of the stack and the PPI. Dimensions, such as the thickness of the liquid metal heat sink, as well as the surface area of its top and bottom copper plates, are therefore based on the requirements constrained by the stack and PPI geometries. Fig. 6 shows the principle of operation of the proposed heat sink. The collector current $\left(\mathrm{I}_{\mathrm{C}}\right)$ drives the liquid metal coolant in the presence of a magnetic field provided by permanent magnets. Each side of the heat sink contains a plate that its inner 


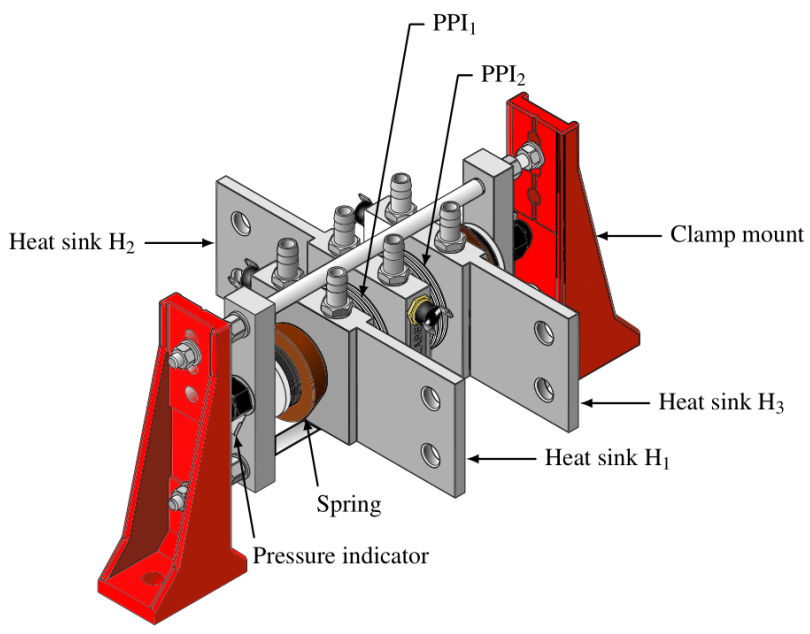

Fig. 5. Schematic of press-pack stack.

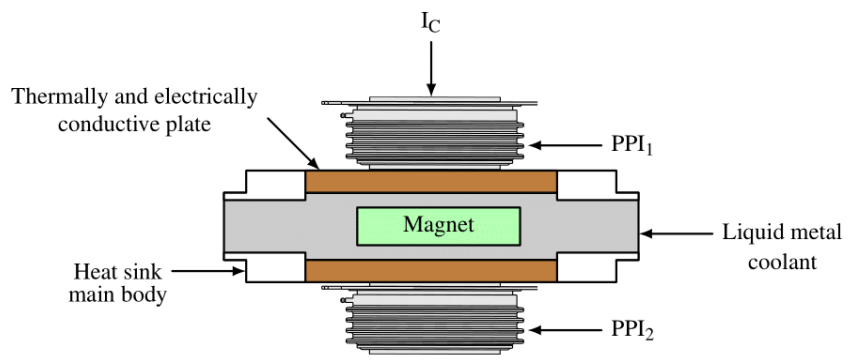

Fig. 6. Schematic of proposed heat sink.

TABLE I

THERMOPHYSICAL PROPERTIES OF LIQUID METAL AND WATER

\begin{tabular}{lccc}
\hline \hline \multicolumn{1}{c}{ Property } & Unit & Water & $\mathrm{Ga}_{68} \mathrm{In}_{22} \mathrm{Sn}_{10}$ \\
\hline Density & $\mathrm{kg} / \mathrm{m}^{3}$ & 998 & 6400 \\
Melting point & ${ }^{\circ} \mathrm{C}$ & 0 & -19 \\
Boiling point & ${ }^{\circ} \mathrm{C}$ & 100 & $>1300$ \\
Specific heat capacity & $\mathrm{J} /\left(\mathrm{kg} \cdot{ }^{\circ} \mathrm{C}\right)$ & 4181 & 365 \\
Dynamic viscosity & $\mathrm{Pa} \cdot \mathrm{s}$ & 0.001 & 0.0024 \\
Electrical conductivity & $\mathrm{S} / \mathrm{m}$ & $5.5 \cdot 10^{-6}$ & $3.46 \cdot 10^{6}$ \\
Thermal conductivity & $\mathrm{W} /\left(\mathrm{m} \cdot{ }^{\circ} \mathrm{C}\right)$ & 0.606 & 16.5 \\
Prandtl number & - & 6.62 & 0.027 \\
\hline \hline
\end{tabular}

and outer surfaces come in direct contact with the PPI and the liquid metal coolant, respectively. Therefore, the excess heat generated by the PPIs is transferred via the liquid metal. The material selection for the heat sink components are based on their chemical compatibility with the selected liquid metal, $\mathrm{Ga}_{68} \mathrm{In}_{22} \mathrm{Sn}_{10}$ (68\% gallium, $22 \%$ indium and $10 \%$ tin in weight). In addition to that, the top and bottom plates of the heat sink should be a good electrical and thermal conductor. On the other hand, the material used for the main body of the heat sink should be an electrical insulator, thus ensuring that $\mathrm{I}_{\mathrm{C}}$ conducts solely through the liquid metal. The thermophysical properties of $\mathrm{Ga}_{68} \mathrm{In}_{22} \mathrm{Sn}_{10}$ and water, which is a common cooling medium, are shown in Table I.

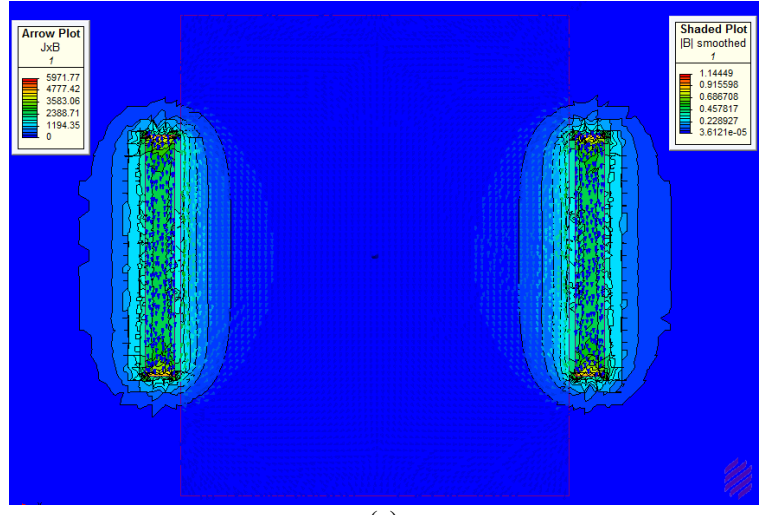

(a)

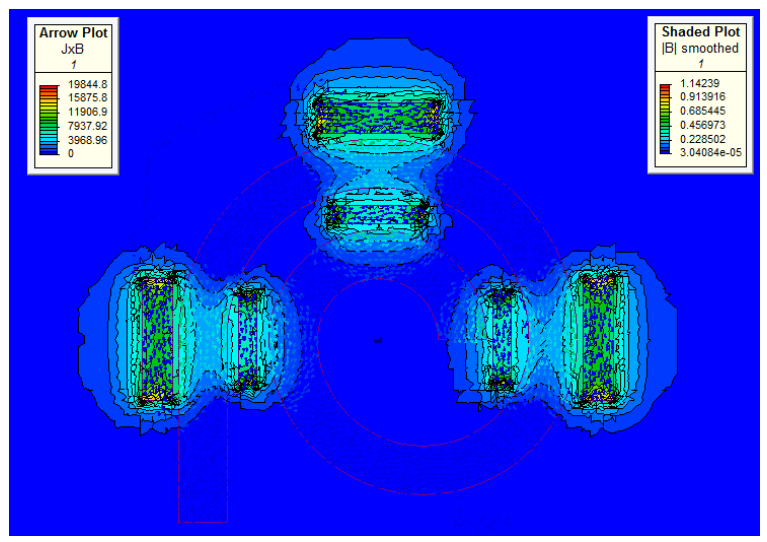

(b)

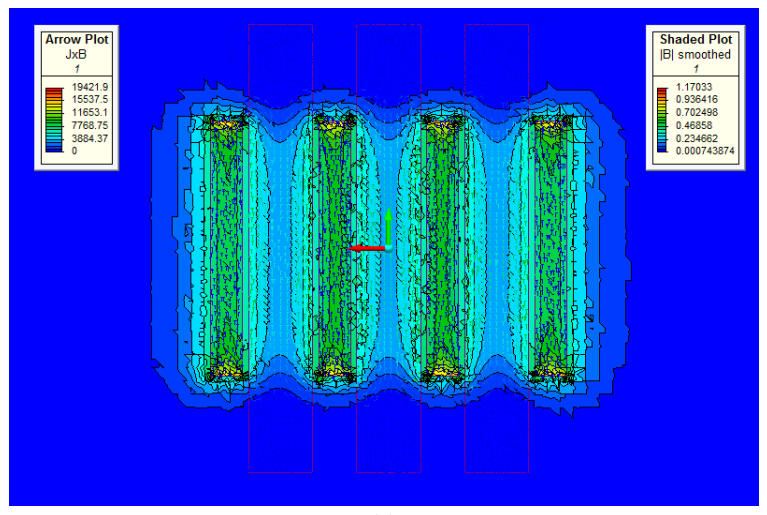

(c)

Fig. 7. Magnetostatic simulation results for various pump geometries: (a) design $\mathrm{A}$ - single channel, (b) design $\mathrm{B}$ - spiral and (c) design $\mathrm{C}$ - parallel channel.

\section{A. Magnetostatic simulation of liquid metal heat sink}

Infolytica MagNet was used to perform various magnetostatic simulations for the design of the liquid metal heat sink, which are presented in this section. Firstly, a magnetostatic simulation is performed for various pump geometries, where 50 A conducting current and N42 Neodymium magnets are used for all simulation models. The results are shown in Fig. 7.

Design A has a large liquid metal channel covering the whole copper lid area and the magnetic field is provided by two magnets placed on each side of the channel. This design is not suitable, as the distance between the two magnets is large and 


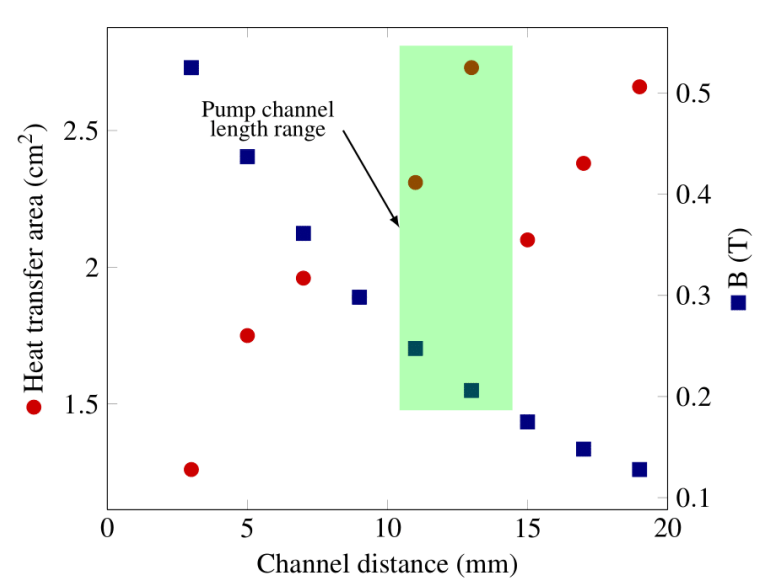

Fig. 8. Magnetostatic analysis of the channel distance in relationship with the magnetic field strength and heat transfer area.

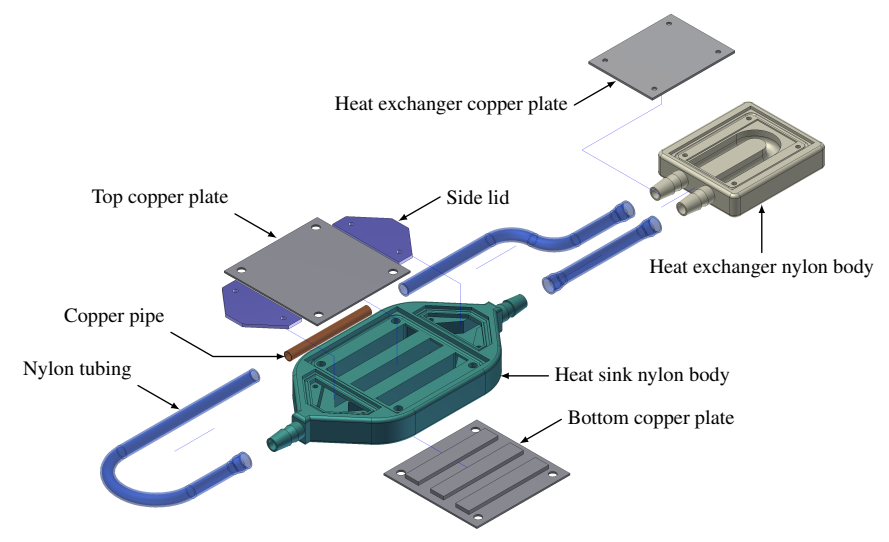

Fig. 9. Exploded view of press-pack heat sink.

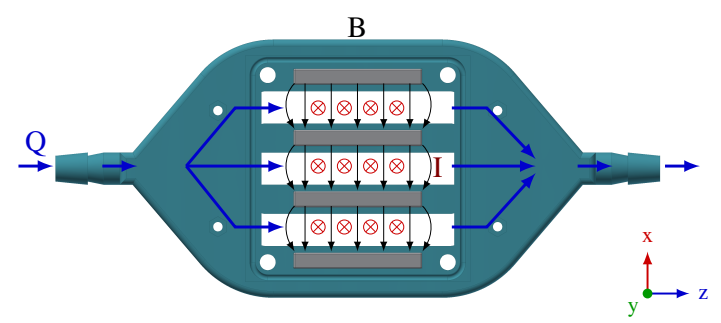

Fig. 10. Working principle of liquid metal heat sink for press-pack devices.

therefore, there is no uniform magnetic field cutting through the conductive coolant. As shown, a Lorentz force is generated only at the sides of the liquid metal channel, thus, there is no fluid flow at the center of the channel. On the other hand, design B shows a spiral structure for the liquid metal, where three sets of magnets are placed for generating the liquid metal flow. Although a large heat transfer area is achieved with the spiral design, the magnets size and position are limited, which impacts the net Lorentz force. Moreover, the implementation of such design is challenging compared to the other two candidates. Finally, design $\mathrm{C}$ proposes a heat sink consisting of parallel channels. A magnetic field is provided through permanent magnets at each channel. The advantage of design $\mathrm{C}$ is that the length of the magnets is not restricted by the channel geometry. Hence, long magnets that are able to cover the majority of the
TABLE II

PHYSICAL PARAMETERS OF COPPER AND NICKEL

\begin{tabular}{lccc}
\hline \hline \multicolumn{1}{c}{ Parameter } & Unit & Copper & Nickel \\
\hline Electrical conductivity & $\mathrm{S} / \mathrm{m}$ & $5.96 \cdot 10^{7}$ & $1.43 \cdot 10^{7}$ \\
Thermal conductivity & $\mathrm{W} /(\mathrm{m} \cdot \mathrm{K})$ & 385 & 90 \\
Relative permeability & - & 0.999994 & $100-600$ \\
\hline \hline
\end{tabular}

current path can be used. In addition, the channel length (i.e. the distance between the magnets) can be altered, so as sufficient magnetic field strength is provided for the pump. As shown, the largest Lorentz force is generated with design C. Therefore, a heat sink that features parallel channels is selected for this work.

The relationship between the channel length, the magnetic field strength and the heat transfer area is also studied numerically. The geometry of the permanent magnets is maintained constant at $40 \times 10 \times 5 \mathrm{~mm}$, which is the same size as the magnets used in the experimental work. Similarly, the total heat sink area is restricted to $70 \times 70 \mathrm{~mm}$ due to the stack and PPI geometries. Therefore, the heat transfer area of the cooling device and the magnetic field in the midplane of the channel is a function of the channel distance. The results are shown in Fig. 8. For a distance less than $10 \mathrm{~mm}$ there is a strong magnetic field, however the heat transfer area is not large, whereas, for a distance larger than $14 \mathrm{~mm}$ the magnetic field is not sufficient to pump the liquid metal and the effective heat transfer area is reduced, as the number of parallel channels is decreased. A channel distance of $14 \mathrm{~mm}$ is selected in this work, as it provides the largest heat transfer area without compromising the liquid metal pump performance.

\section{B. Design parameters of the liquid metal heat sink}

Fig. 9 illustrates a detailed view of the cooling system for one side of the PPI. As shown, the collector and emitter lids of each PPI come in direct contact with the copper plates of the heat sink, which forms a closed loop with a heat exchanger, whereas the magnets are inserted in pockets between the liquid metal channel. The system is filled with the liquid metal $\mathrm{Ga}_{68} \mathrm{In}_{22} \mathrm{Sn}_{10}$ which transfers heat to the heat exchanger, where it is then dissipated to the ambient through water cold plates that are attached to the heat exchanger copper surface. An intermediate layer made of ceramic aluminum oxide is applied between the heat exchanger and the water cold plate, which provides electrical isolation between the two surfaces while allowing the heat to travel. A cavity exists at the edges of each side lid and copper plate, which is filled with blue silicone gasket and seals the heat sink body. To ensure that air bubbles are removed from the system, an inlet tube is placed in the cooling loop, that has is higher than the main loop, thus forcing the air bubble to exit the system. Fig. 10 shows the operating principle of the liquid metal pump. As the body of the heat sink is made of nylon, the collector current $\mathrm{I}_{\mathrm{C}}$ conducts only through the liquid metal in $y$ plane, which in combination with the constant magnetic field in $x$ plane generates a Lorentz force in $z$ plane that drives the liquid metal. Therefore, the liquid metal pump is self-driven, as no external power is required.

The material selection for the cooling system was performed 


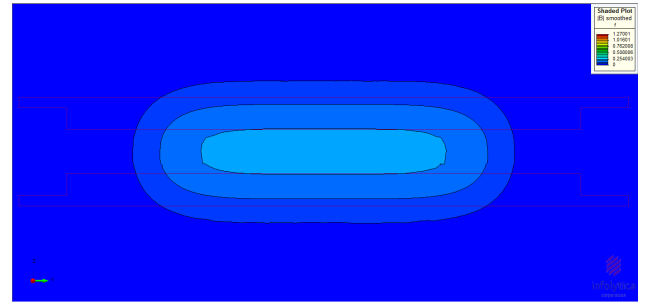

(a)

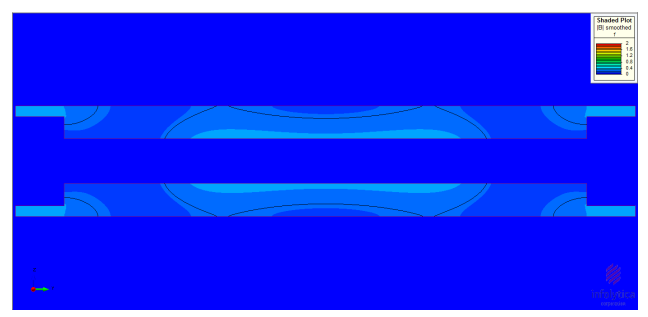

(b)

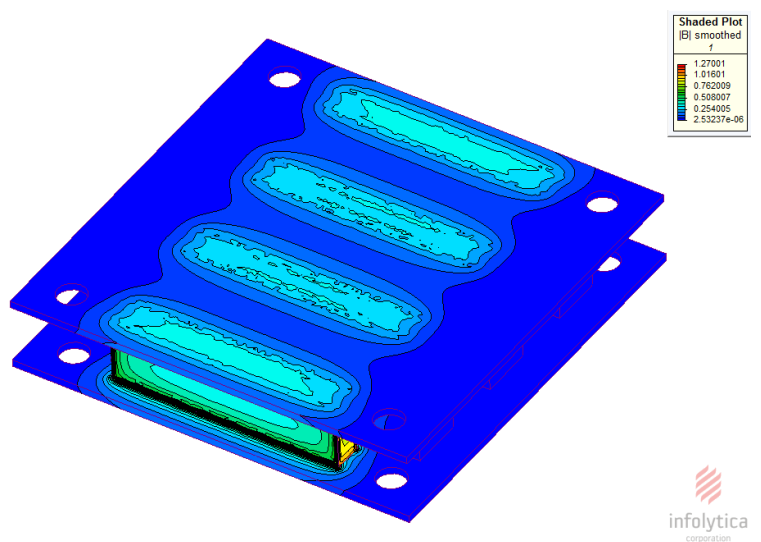

(c)

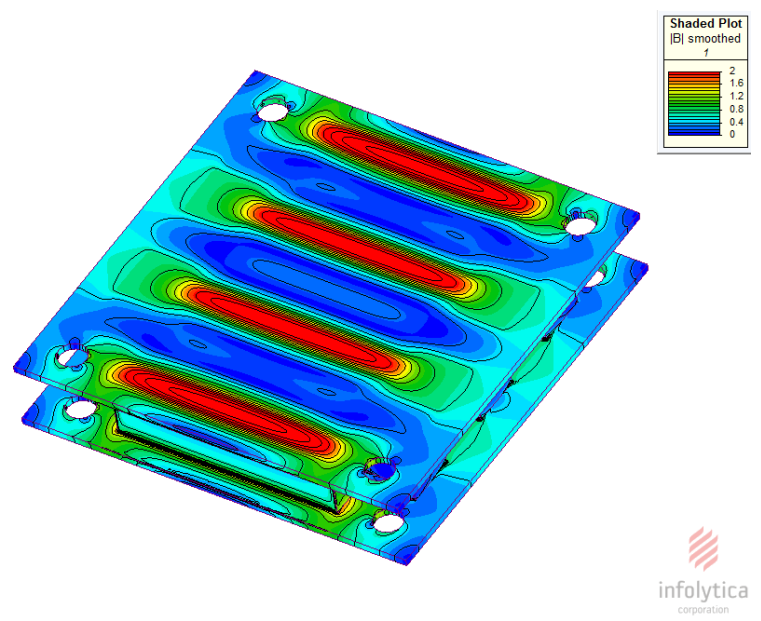

(d)

Fig. 11. Simulation results of magnetic field in the liquid metal pump: magnetic field contour in the active channel of the pump for (a) nickel plate (b) and copper plate. Magnetic leakage for (c) nickel plate and (d) copper plate.

based on the project requirements. The bodies of the heat sink and heat exchanger are 3-D printed using VeroWhite, which is chemically compatible with $\mathrm{Ga}_{68} \mathrm{In}_{22} \mathrm{Sn}_{10}$. VeroWhite is an electrical insulator. Similarly, chemically resistant flexible plastic tubing is used to connect the heat sink to the heat exchanger. Although copper forms a thin oxidation layer when

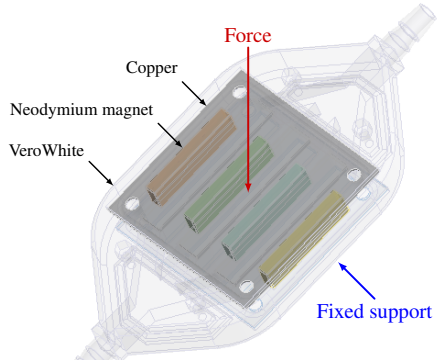

(a)

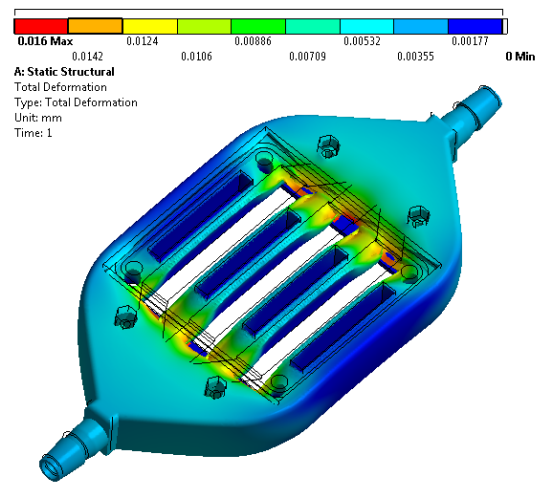

(b)

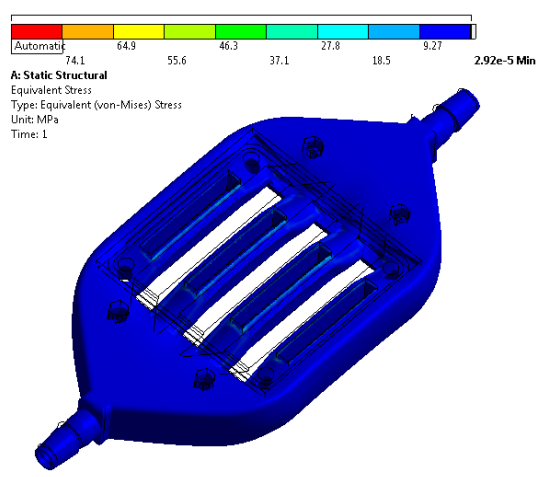

(c)

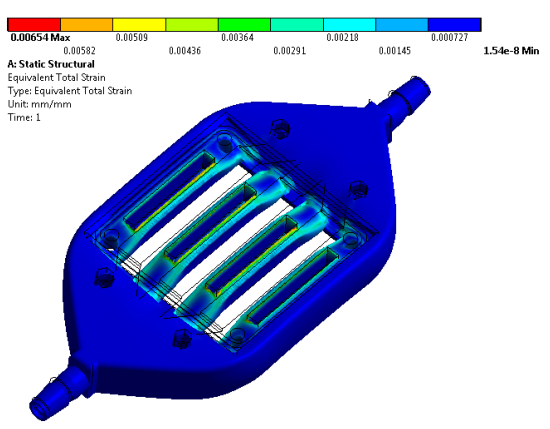

(d)

Fig. 12. Simulation results of static structural analysis for the heat sink: (a) simulation model (b) total deformation (c) equivalent stress and (d) equivalent strain.

it is in direct contact with $\mathrm{Ga}_{68} \mathrm{In}_{22} \mathrm{Sn}_{10}$, its mechanical rigidity and long-term reliability are not compromised. Nonetheless, 
applying a thin nickel layer $(0.02 \mathrm{~mm})$ ensures protection against corrosion. Alternatively, pure nickel was considered for the lids, as it does not corrode against $\mathrm{Ga}_{68} \mathrm{In}_{22} \mathrm{Sn}_{10}$. However, as shown in Table II, copper has significantly superior thermal and electrical properties. In addition, nickel has very high relative magnetic permeability $\left(\mu / \mu_{0}\right)$, thus affecting the magnetic field in the active channel of the pump. The effect of copper and nickel lids on the magnetic field was investigated numerically. As observed from Fig. 11, there is a uniform magnetic field of $0.2 \mathrm{~T}$ by using copper plates. Since the relative permeability of the copper is close to 1 , it behaves similarly to air and does not distort or interfere with the magnetic field. Therefore, magnetic leakage does not occur (Fig. 11(c)) and the magnetic field cuts through the liquid metal. On the other hand, using nickel results in a magnetic field of $0 \mathrm{~T}$ through the liquid metal, since there is a large magnetic flux leakage caused by the nickel plates. In the simulation model the best-case scenario of $\mu / \mu_{0}=100$ was selected for nickel. The case where a nickel plated copper plate was also investigated, without yielding any variations compared to the pure copper model. Another disadvantage of nickel compared to copper is its higher cost. The press-pack devices were tested for switching frequencies up to $1 \mathrm{kHz}$, without any interference caused by the magnetic field.

\section{Static structural analysis of the heat sink}

The cooling system is constantly subjected to a high clamping force; therefore, a static structural analysis was performed in ANSYS Mechanical to evaluate the stress and strain magnitude within the heat sink structure and to determine whether a plastic strain will occur in the event of excessive pressure being applied. A maximum force of $10 \mathrm{kN}$ is applied at the top copper lid, whereas the bottom copper lid is configured as a fixed support. The value of $10 \mathrm{kN}$ was selected based on the recommendation of the press-pack manufacturer regarding the maximum pressure applied on the stack. Moreover, all the contact connections between different bodies were configured as rough. As the results in Fig. 12 show, the maximum strain is observed at the edges of the heat sink body, however, it is only a few $\mu \mathrm{m}$. That is because the edges of the plastic housing are the main contact surfaces between the two copper plates. Nonetheless, the elastic deformation is minimum and does not compromise the functionality of the heat sink. Also, no plastic strain is observed, which could lead to failure of the cooling system. Thus, the nylon prototype heat sinks can withstand the required static pressure for the stack for the experimental validation of the cooling system. However, during the experimental testing the glass transition temperature $(T \mathrm{~g})$ of VeroWhite, which equals $52^{\circ} \mathrm{C}$, should not be exceeded, as its physical properties are altered at that point.

\section{Experimental Evaluation for Press-Pack Heat SinK}

\section{A. Experimental setup for heat sink evaluation}

The steady state heat transfer capability of the liquid metal heat sink is experimentally evaluated through a series of tests,

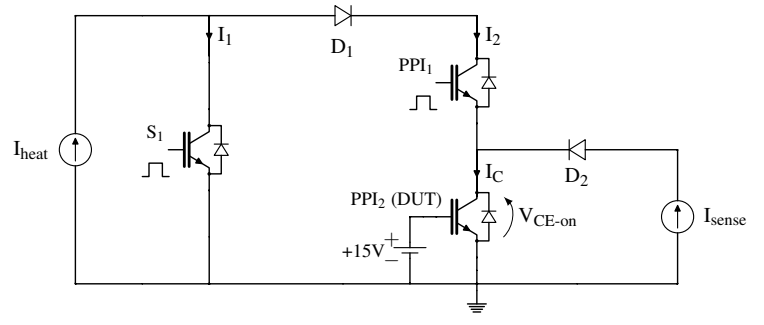

Fig. 13. Schematic diagram of PPI heating and temperature sensing.

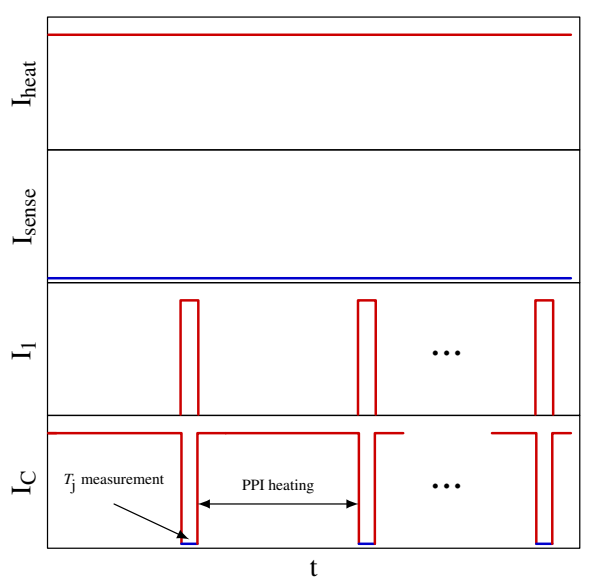

(a)

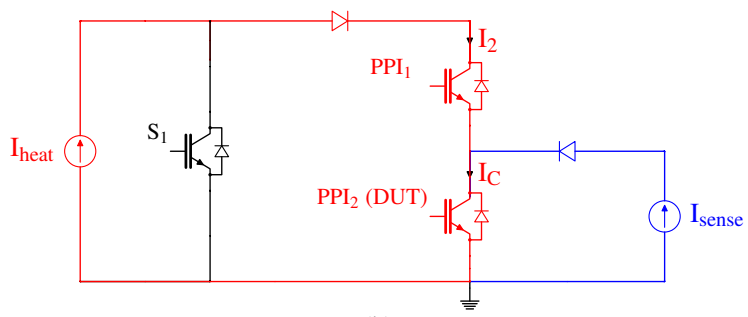

(b)

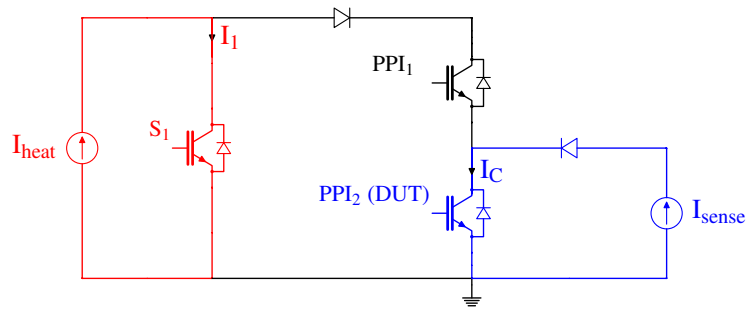

(c)

Fig. 14. PPI heating and temperature measurement: (a) example waveform for PPI heating and $T_{\mathrm{j}}$ measurement, (b) heating phase and (c) temperature measurement phase.

in which the water heat sinks $\mathrm{H} 2$ and $\mathrm{H} 3$ (Fig. 5) are replaced by the proposed heat sinks. The heat load of the system is generated by the self-heating of the PPI devices due to the power losses, $\mathrm{P}(\mathrm{t})$. The schematic diagram of the circuit used for heating the PPI device and sensing the junction temperature, $T_{\mathrm{j}}$, is shown in Fig. 13. Devices $\mathrm{PPI}_{1}$ and $\mathrm{PPI}_{2}$ are press-pack devices mounted on the same stack, as illustrated in Fig. 5. The TopCon Quadro programmable power supply unit provides a constant DC current, Iheat, for heating the DUT, whereas, Isense provides a small current for temperature sensing. Isense causes a voltage drop across the IGBT chip which is a Temperature 


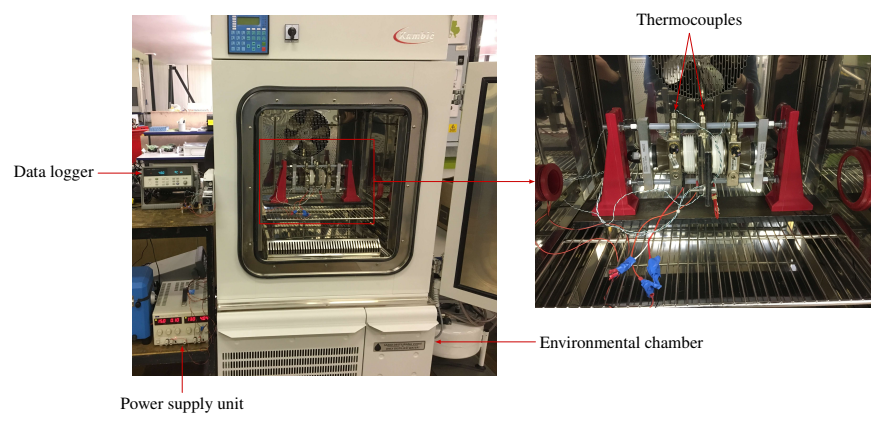

Fig. 15. TSEP calibration test setup.

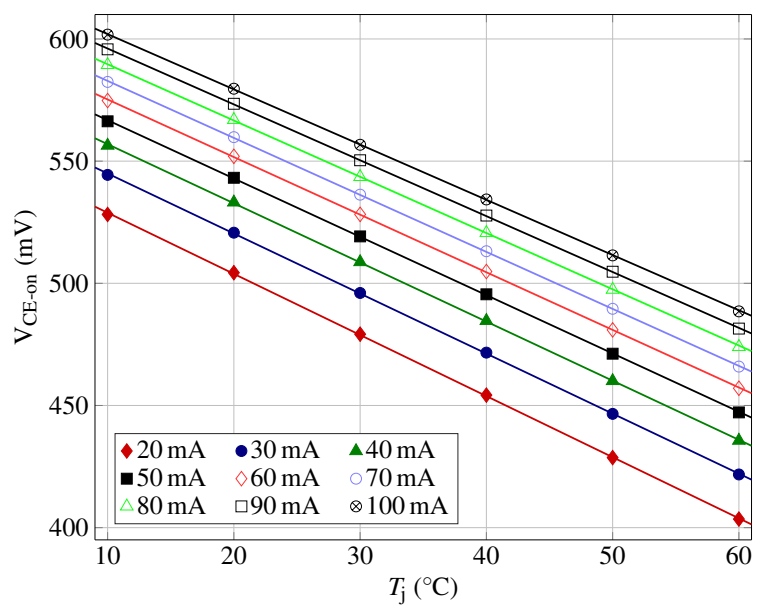

Fig. 16. TSEP calibration curves of PPI for various sense currents.

Sensitive Electric Parameter (TSEP). Diodes $\mathrm{D}_{1}$ and $\mathrm{D}_{2}$ are placed in order to force $\mathrm{I}_{\text {sense }}$ and Iheat to flow through $\mathrm{PPI}_{2}$, which is the DUT. The IGBT switch $\mathrm{S}_{1}$ serves as auxiliary switch and provides a path to $\mathrm{I}_{\text {heat }}$ whenever PPI $\mathrm{I}_{1}$ is switched off.

The current waveforms for heating the IGBT and $T_{\mathrm{j}}$ sensing is shown in Fig. 14. The DUT remains in its forward conducting state for the whole thermal characterization process, by applying $+15 \mathrm{~V}$ across the gate and emitter. During the heating phase, $\mathrm{S}_{1}$ is switched off while $\mathrm{PPI}_{1}$ is conducting, thus allowing $\mathrm{I}_{\text {heat }}$ to also conduct through $\mathrm{PPI}_{2}$. On the other hand, during the temperature measurement process $\mathrm{S}_{1}$ conducts while $\mathrm{PPI}_{1}$ is switched off. At that instance only $\mathrm{I}_{\text {sense }}$ is conducting through $\mathrm{PPI}_{2}$, therefore, the TSEP is applied. The DUT is heated for 98.5 $\mathrm{ms}$, whereas, the temperature measurement process lasts for 1.5 ms. There is an overlap of a few $\mu$ s, during which both $S_{1}$ and $\mathrm{PPI}_{2}$ are conducting simultaneously, in order to prevent the $\mathrm{I}_{\text {heat }}$ power supply switching to constant voltage mode.

The dependency of $\mathrm{V}_{\text {CE-on }}$ on $T_{\mathrm{j}}$ for a low conducting current was selected for sensing the junction temperature of the DUT. Unlike the IGBT devices that are housed in a conventional package, press-pack devices require a pressing force that ensures electrical and thermal contact. Therefore, the calibration of the TSEP cannot be performed using a temperature-controlled plate [43], as the device should be pressed and heated concurrently. Instead, an environmental chamber is used, where the press-pack stack is heated uniformly. The DUT is constantly in its forward conducting

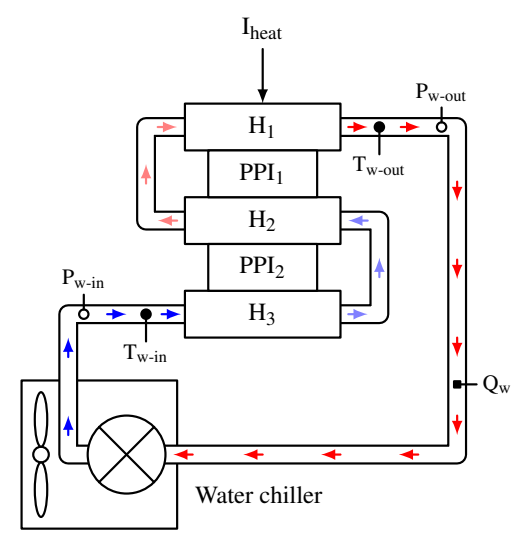

(a)

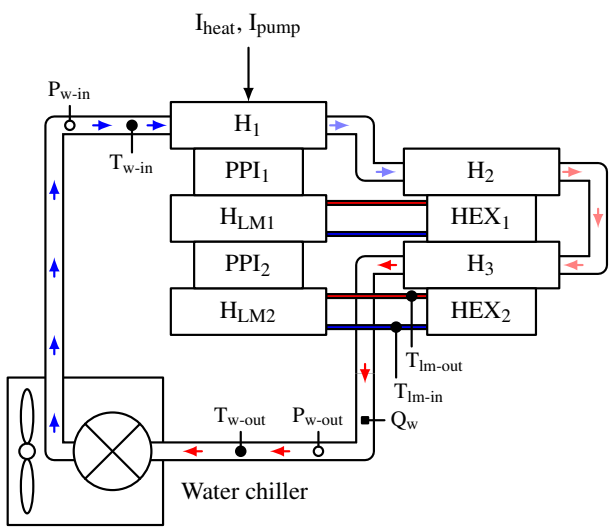

(b)

Fig. 17. Schematic diagram of press-pack cooling circuit: (a) water cooling system and (b) liquid metal cooling system with liquid metal-to-water heat exchangers.

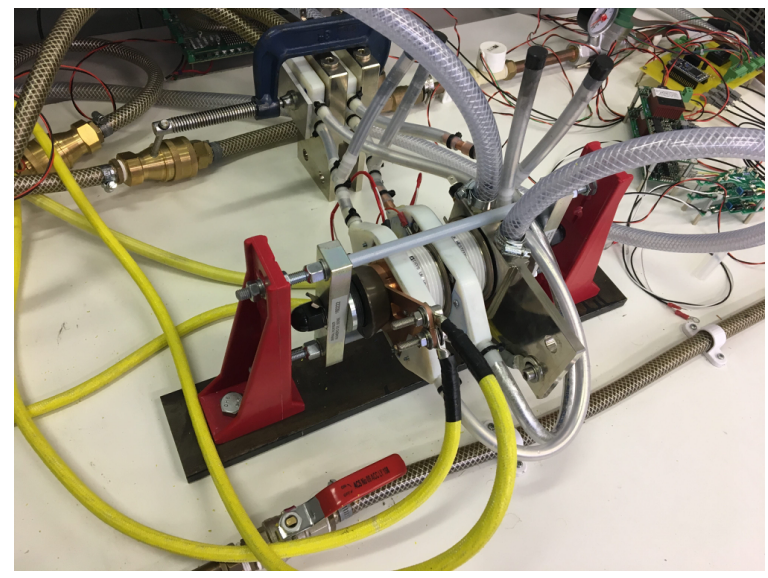

Fig. 18: Experimental test setup for press-pack cooling using the prototype liquid metal cooling system with liquid metal-to-water heat exchangers.

state and Isense conducts through it for the whole duration of the calibration. The temperature of various locations was monitored with thermocouples that were mounted on the heat sink bodies and inside the hollow heat sink pipes. The TSEP calibration setup is shown in Fig. 15. Each $\mathrm{V}_{\mathrm{CE}-\text { on }}$ reading is taken once the DUT is in thermal equilibrium (i.e. once all thermocouples sense the same temperature). The $\mathrm{V}_{\mathrm{CE}-\mathrm{on}}$ is recorded for $\mathrm{I}_{\text {sense }}$ between $20-100 \mathrm{~mA}$ in $10 \mathrm{~mA}$ intervals. The results of the TSEP calibration process are presented in Fig. 16. The value of $20 \mathrm{~mA}$ was selected as sense current for 


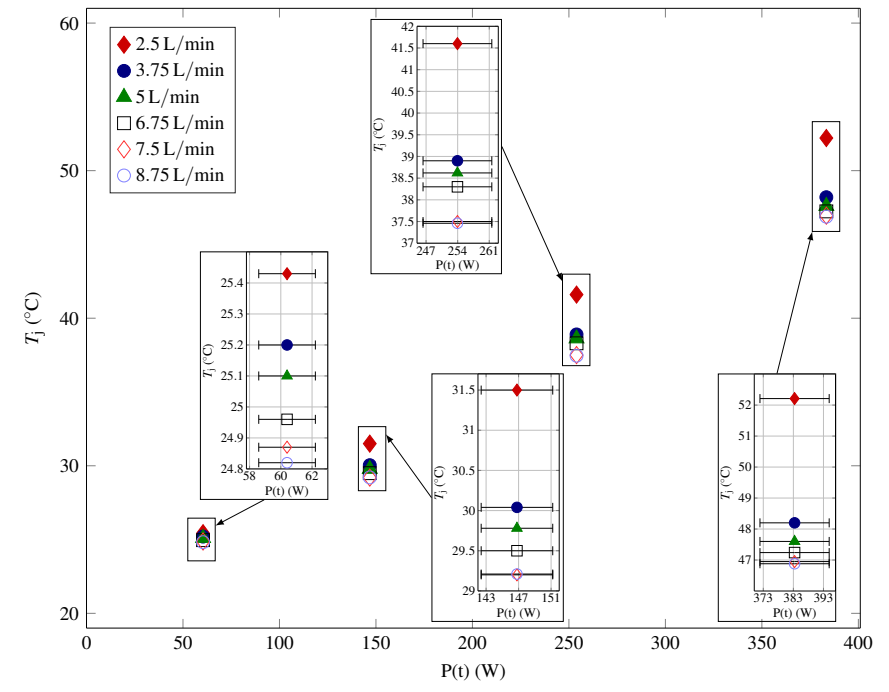

Fig. 19. Experimental results for water press-pack cooling system under various heat loads and flow rates.

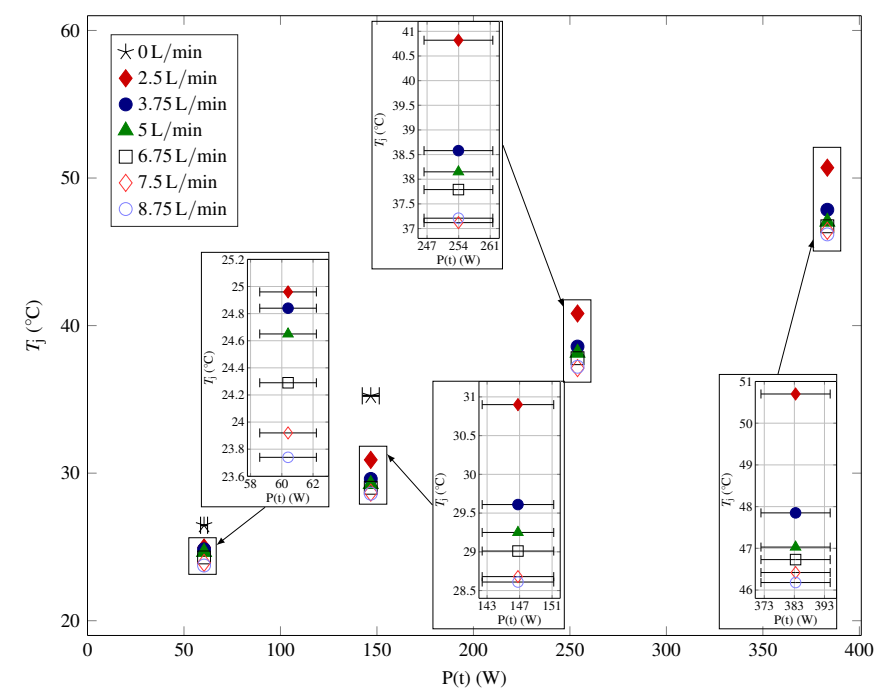

Fig. 20. Experimental results for liquid metal press-pack cooling system under various heat loads and flow rates.

temperature measurement of the PPI device, as it provides the highest resolution $\left(-2.5 \mathrm{mV} /{ }^{\circ} \mathrm{C}\right)$. In addition, the $\mathrm{R}^{2}$ value of the graph approaches 1 , thus indicating a very good linear regression of the curve. Hence, for a constant sense current of $20 \mathrm{~mA}$ and a given $\mathrm{V}_{\mathrm{CE}-\mathrm{on}}(\mathrm{V})$, the junction temperature, $T_{\mathrm{j}}\left({ }^{\circ} \mathrm{C}\right)$, is defined as:

$$
T_{\mathrm{j}}=221.47-400 \cdot \mathrm{V}_{\mathrm{CE}-\mathrm{on}}
$$

\section{B. Experimental results of press-pack heat sink}

The heat dissipation performance of the liquid metal heat sink is evaluated through a steady state heat transfer experimental test and is compared against a conventional water-based commercial heat sink for PPIs. The schematics of the cooling circuits for water and liquid metal heat sinks are presented in Fig. 17 (a) and (b), respectively. In both tests, a flow meter $\left(\mathrm{Q}_{\mathrm{w}}\right)$, pressure transducers $\left(\mathrm{P}_{\mathrm{w}-\text { in }}\right.$ and $\left.\mathrm{P}_{\mathrm{w}-\mathrm{out}}\right)$, and thermocouples $\left(\mathrm{T}_{\mathrm{w} \text {-in }}\right.$ and $\left.\mathrm{T}_{\mathrm{w} \text {-out }}\right)$ are used for monitoring the water flow rate, the pressure drop across the water cooling loop and the inlet and outlet temperatures of water. Additional

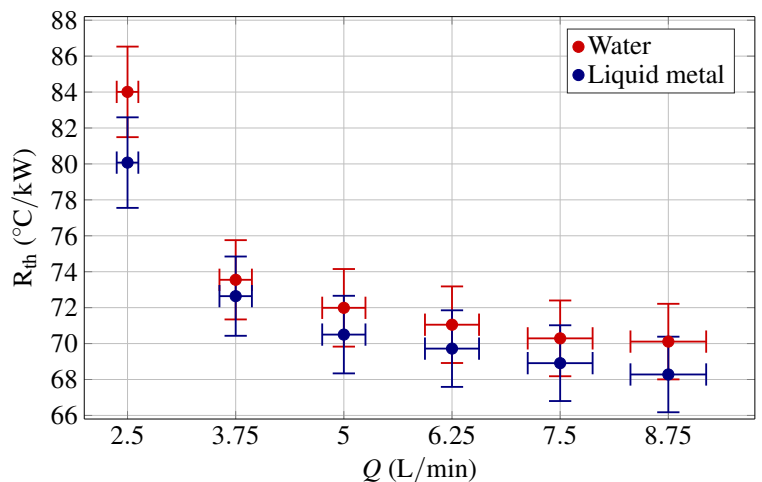

Fig. 21. Thermal resistance of cooling systems as a function of the water flow rate.

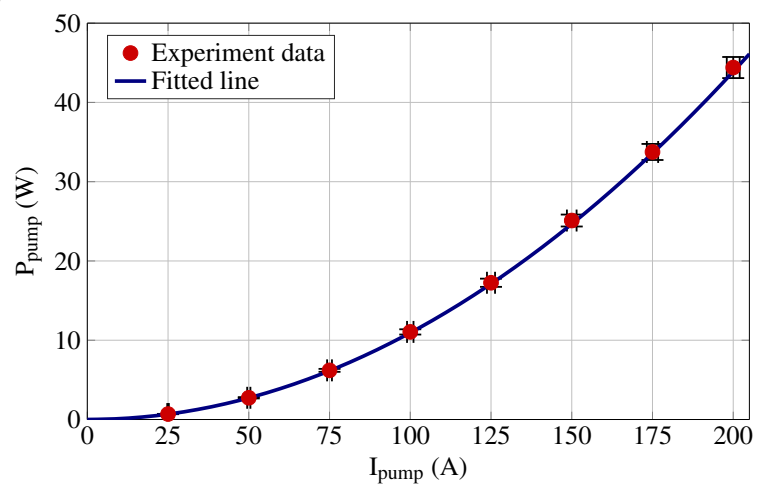

Fig. 22. Consumption power of pump as a function of the input current.

thermocouples ( $\mathrm{T}_{\mathrm{lm}-\mathrm{in}}$ and $\left.\mathrm{T}_{\mathrm{lm} \text {-out }}\right)$ are placed for monitoring the inlet and outlet temperature of the liquid metal loop. Fig. 18 shows a picture of the proposed liquid metal cooling system.

The results of the thermal characterization for water and liquid metal cooling schemes are shown in Fig. 19 and 20, respectively. The two cooling methods were subjected to a heating current, Ineat, ranging from 50 to $200 \mathrm{~A}$, which corresponds to heat loads between 60 and $384 \mathrm{~W}$. In addition, the flow rate of the water cooling circuit was varied throughout the experimental process. Each measurement is performed after the DUT has been heated for 30 minutes, whereas the water inlet temperature is fixed at $20^{\circ} \mathrm{C}$. As observed, the cooling effectiveness of both cooling systems is reduced as the coolant's flow rate decreases. A special case where the water cooling system is shut off and thus emulates a water pump failure is also tested and presented in Fig. 19. For that part of the experiment, the water chiller is switched off, resulting in a net water flow rate of $0 \mathrm{l} / \mathrm{min}$. Therefore, the heat is transported through the liquid metal to the heat exchanger and then is dissipated to the ambient via the copper bodies of the water heat sinks.

The thermal resistance of the cooling system for various flow rates is derived from the experimental data presented in Fig. 19 and 20 and is shown in Fig. 21. As demonstrated, the proposed liquid metal cooling system provides lower thermal resistance for identical water flow rates. Thus, higher heat loads can be rejected without the risk of thermal runaway. Based on the results one can conclude that the proposed method offers higher cooling capability and electrically isolated cooling mechanisms that eliminate the problem associated with corrosion in HVDC 
systems, has no moving parts and does not require any external power source. As the direction of the magnetic field is fixed, inverting the conducting current would reverse the liquid metal flow, thus resulting in oscillating movement of the coolant. As such the proposed scheme applies to press-packs that have IGBT chips only without anti-parallel diodes. In HVDC applications IGBT chips only press-packs are common [44]. Finally, Fig. 22 shows the consumption power of the pump as a function of the input current. This adds approximately an additional $10 \%$ to the heat generated by the IGBT device at the maximum tested power. As the purpose of the project was to develop a liquid metal heat sink that is self-driven the additional heat is a by-product of the design. One way to avoid the additional heat generated in the liquid metal is to use a copper bus bar that overlaps the heat sink and provides a path for the current, while using an external MHD pump. An additional power supply would be needed for the MHD pump in that case. A hybrid solution would be to use a material for the bus bar that has an electrical conductivity that is close to the liquid metal, thus creating several paths for the current and limiting the current passing through the liquid metal. However, in that case additional losses will be created as well.

\section{CONCLUSION}

PPI modules have significant advantages over conventional modules, such as the ability to apply double-side cooling and higher tolerance against large temperature fluctuations because of its internal structure. Nonetheless, their use in HVDC power stations in combination to a water-based cooling system leads to overcomplicated cooling networks. Moreover, there is a risk of failure of the cooling system due to piping corrosion as a product of the interaction between the conducting current and the coolant. This paper has presented a heat sink based on a liquid metal agent for PPI devices. Heat is transported from the PPI device via convection to the liquid metal coolant, which is driven by an integrated MHD pump, to a liquid metal-to-water heat exchanger. Each liquid metal cooling loop is electrically isolated to their adjacent loops, therefore, the same water loop can be used without risking current conducting through water and thus leading to the development of corrosion in the water piping system. In addition, the load current conducting through PPIs is used for driving the liquid metal, hence, the liquid metal cooling system is self-driven and does not depend on an external power source. Experimental comparisons between the liquid metal-based cooling system and a commercial waterbased cooling system have demonstrated a junction temperature reduction under identical working conditions by employing the proposed cooling scheme.

\section{REFERENCES}

[1] C. Qian et al., "Thermal Management on IGBT Power Electronic Devices and Modules," in IEEE Access, vol. 6, pp. 12868-12884, 2018.

[2] B. Ji, V. Pickert, B. Zahawi, and M. Zhang, "In-situ bond wire health monitoring circuit for IGBT power modules," in Power Electronics, Machines and Drives (PEMD 2012), 6th IET International Conference on, Conference Proceedings, pp. 1-6.

[3] B. Ji, X. Song, W. Cao, V. Pickert, J. Hu, J. W. Mackersie, and G. Pierce, "In situ diagnostics and prognostics of solder fatigue in IGBT modules for electric vehicle drives," Power Electronics, IEEE Transactions on, vol. 30, no. 3, pp. 1535-1543, 2015.

[4] A. Qawasmi, J. Teichrib, N. Venkatesh and R. W. De Doncker, "A New Thyristor-Based Power Electronic Device for DC Circuit Breakers in Medium-Voltage Applications," 2018 9th IEEE International Symposium on Power Electronics for Distributed Generation Systems (PEDG), Charlotte, NC, 2018, pp. 1-6.

[5] E. Deng, Z. Zhao, Z. Lin, R. Han, and Y. Huang, "Influence of temperature on the pressure distribution within press pack igbts," IEEE Transactions on Power Electronics, vol. 33, no. 7, pp. 6048-6059, July 2018.

[6] Qin, R., Du, Y., Jiang, Z., Wang, X., Fu, A. and Lu, Y., 2018. Corrosion Behavior of X80 Pipe Steel under HVDC Interference in Sandy Soil. Metals, 8(10), p.809.

[7] Y. Deng and J. Liu, "Hybrid liquid metal-water cooling system for heat dissipation of high power density microdevices," Heat and Mass Transfer, vol. 46, no. 11, pp. 1327-1334, Dec 2010.

[8] Y. Hayashi, N. Saneie, Y. J. Kim, and J.-H. Kim, "Thermal performance and pressure drop of galinstan-based microchannel heat-sink for high heat-flux thermal management," no. 56871, p. V001T07A012, 2015, 10.1115/ICNMM2015-48404.

[9] M. Hodes, R. Zhang, L. S. Lam, R. Wilcoxon, and N. Lower, "On the potential of galinstan-based minichannel and minigap cooling," IEEE Transactions on Components, Packaging and Manufacturing Technology, vol. 4, no. 1, pp. 46-56, Jan 2014.

[10] R. Zhang, M. Hodes, N. Lower, and R. Wilcoxon, "Water-based microchannel and galinstan-based minichannel cooling beyond $1 \mathrm{~kW} / \mathrm{cm} 2$ heat flux," IEEE Transactions on Components, Packaging and Manufacturing Technology, vol. 5, no. 6, pp. 762-770, June 2015.

[11] S. Wang, Z. Song, P. Fu, K. Wang, X. Xu, W. Tong, and Z. Wang, "Thermal analysis of water-cooled heat sink for solid-state circuit breaker based on igcts in parallel," IEEE Transactions on Components, Packaging and Manufacturing Technology, pp. 1-1, 2018.

[12] S. Fang, W. Luo, H. Wang, and H. Hu, "Operational performance of the valve cooling system in Guangzhou converter station," in 11th IET International Conference on AC and DC Power Transmission, Feb 2015, pp. $1-5$.

[13] ABB, "Swedewater Cooling Systems Solutions for reliable energy supply", technical report, 2016.

[14] J.-S. Kwak, C.-K. Kim, and B.-E. Koh, "Cooling system of Haenam-Jeju HVDC system [power convertor]," in ISIE 2001. 2001 IEEE International Symposium on Industrial Electronics Proceedings (Cat. No.01TH8570), vol. 2, June 2001, pp. 1000-1005 vol.2.

[15] X. Song, Y. Cheng, B. Gao, Y. Fan, T. He and J. Ran, "Sediment deposition model of grading electrode in water cooling circuit of HVDC converter valve," in The Journal of Engineering, vol. 2019, no. 16, pp. 3396-3401, 32019.

[16] Geng M, Lu Z. The Application of PVDF in Converter Cooling Pipeline. InIOP Conference Series: Earth and Environmental Science 2017 Nov (Vol. 94, No. 1, p. 012135).

[17] Habert, M., and B. Agostini. "Air-Cooled Thermosyphon for Press-Pack Stack of Semiconductors." Journal of Physics Conference Series. Vol. 745. No. 3. 2016.

[18] M. Haghparast, "Transient analysis of magnetohydrodynamic seawater thrusters with decaying magnetic field," Ships and Offshore Structures, vol. 12, no. 5, pp. 591-598, 2017.

[19] M. Haghparast, M. R. A. Pahlavani, and D. Azizi, "Numerical investigation of the effects of magnetic field and fluid electrical conductivity on the performance of marine magnetohydrodynamic motors," IET Electric Power Applications, May 2018.

[20] J. Overduin, V. Polyak, A. Rutah, T. Sebastian, J. Selway, and D. Zile, "The Hunt for Red October II: A magnetohydrodynamic boat demonstration for introductory physics," The Physics Teacher, vol. 55, no. 8, pp. 460-466, 2017.

[21] S. Khripchenko, S. Lekomtsev, S. Denisov, V. Dolgikh, and A. Pavlinov, "Laboratory model of the aluminum furnace with mhd stirring induced by a rod-like inductor generating a travelling magnetic field." Magnetohydrodynamics (0024-998X), vol. 53, no. 2, 2017.

[22] K. E. Bolotin, V. E. Frizen, I. F. Sokolov, and S. A. Bychkov, "Numerical simulation of MHD stirrer for 12 ton metallurgical aggregate," in 2018 IEEE Conference of Russian Young Researchers in Electrical and Electronic Engineering (EIConRus), Jan 2018, pp. 585-587.

[23] V. Timofeev and M. Khatsayuk, "Theoretical design fundamentals for MHD stirrers for molten metals." Magnetohydrodynamics (0024-998X), vol. 52 , no. $4,2016$. 
[24] V. Batenin, I. Belyaev, V. Sviridov, E. Sviridov, and Y. I. Listratov, "Modernization of the experimental base for studies of MHD heat exchange at advanced nuclear power facilities," High Temperature, vol. 53 , no. 6, pp. 904-907, 2015.

[25] J. E. Nieminen and C. O. Maidana, "Comparative studies for the MHD modeling of annular linear induction pumps for space applications," in 15th International Energy Conversion Engineering Conference, 2017, p. 4963.

[26] A. Shahidian, M. Ghassemi, S. Khorasanizade, M. Abdollahzade, and G. Ahmadi, "Flow analysis of non-newtonian blood in a magnetohydrodynamic pump," IEEE transactions on magnetics, vol. 45, no. 6, pp. 2667-2670, 2009.

[27] F. Khan, D. Baucom, C. D. Heyes, and I. Fritsch, "Studies toward lab-ona-chip separations and detection using redox magnetohydrodynamic microfluidics," in Meeting Abstracts, no. 42. The Electrochemical Society, 2015, pp. 2227-2227.

[28] U. Ghoshal, D. Grimm, S. Ibrani, C. Johnston, and A. Miner, "Highperformance liquid metal cooling loops," in Semiconductor Thermal Measurement and Management IEEE Twenty First Annual IEEE Symposium, 2005., March 2005, pp. 16-19.

[29] Y. Deng and J. Liu, "Design of practical liquid metal cooling device for heat dissipation of high performance CPUs," Journal of Electronic Packaging, vol. 132, no. 3, pp. 031 009- 031 009-6, 2010, 10.1115/1.4002012.

[30] Y. Deng and J. Liu, "Optimization and evaluation of a high-performance liquid metal CPU cooling product," IEEE Transactions on Components, Packaging and Manufacturing Technology, vol. 3, no. 7, pp. 1171-1177, July 2013.

[31] T. Li, Y.-G. Lv, J. Liu, and Y.-X. Zhou, “A powerful way of cooling computer chip using liquid metal with low melting point as the cooling fluid," Forschung im Ingenieurwesen, vol. 70, no. 4, pp. 243-251, Dec 2005.

[32] Zhang, X.D., Li, X.P., Zhou, Y.X., Yang, J. and Liu, J., 2019. Vascularized liquid metal cooling for thermal management of $\mathrm{kW}$ high power laser diode array. Applied Thermal Engineering, 162, p.114212.

[33] J. Vetrovec, D. A. Copeland, R. Feeler, and J. Junghans, "Testing of active heat sink for advanced high-power laser diodes," Proc.SPIE, vol. 7918, pp. $7918-7918-6,2011$.

[34] Y. Deng and J. Liu, "A liquid metal cooling system for the thermal management of high power LEDs," International Communications in Heat and Mass Transfer, vol. 37, no. 7, pp. 788 - 791, 2010.

[35] M. Luo, Y. Zhou, and J. Liu, "Blade heat dissipator with roomtemperature liquid metal running inside a sheet of hollow chamber," IEEE Transactions on Components, Packaging and Manufacturing Technology, vol. 4, no. 3, pp. 459-464, March 2014.

[36] R.Wilcoxon, N. Lower, and D. Dlouhy, "A compliant thermal spreader with internal liquid metal cooling channels," in 2010 26th Annual IEEE Semiconductor Thermal Measurement and Management Symposium (SEMI-THERM), Feb 2010, pp. 210-216.

[37] A. Sakanova, C. F. Tong, K. J. Tseng, R. Simanjorang, and A. K. Gupta, "Weight consideration of liquid metal cooling technology for power electronics converter in future aircraft," in 2016 IEEE 2nd Annual Southern Power Electronics Conference (SPEC), Dec 2016, pp. 1-5.

[38] Zhang, X.D., Yang, X.H., Zhou, Y.X., Rao, W., Gao, J.Y., Ding, Y.J., Shu, Q.Q. and Liu, J., 2019. Experimental investigation of galinstan based minichannel cooling for high heat flux and large heat power thermal management. Energy conversion and management, 185, pp.248-258.

[39] Amy, C., Budenstein, D., Bagepalli, M., England, D., DeAngelis, F., Wilk, G., Jarrett, C., Kelsall, C., Hirschey, J., Wen, H. and Chavan, A., 2017. Pumping liquid metal at high temperatures up to 1,673 kelvin. Nature, 550(7675), pp.199-203.

[40] M. Tawk, Y. Avenas, A. Kedous-Lebouc, and M. Petit, "Numerical and experimental investigations of the thermal management of power electronics with liquid metal minichannel coolers," IEEE Transactions on Industry Applications, vol. 49, no. 3, pp. 1421-1429, May 2013.

[41] M. Luo and J. Liu, "Experimental investigation of liquid metal alloy based mini-channel heat exchanger for high power electronic devices," Frontiers in Energy, vol. 7, no. 4, pp. 479-486, Dec 2013.

[42] Y. Yerasimou, V. Pickert, B. Ji and X. Song, "Liquid Metal Magnetohydrodynamic Pump for Junction Temperature Control of Power Modules," in IEEE Transactions on Power Electronics, vol. 33, no. 12, pp. 10583-10593, Dec. 2018.

[43] C. Chen et al., "Signal Sweeping Technique to Decouple the Influence of Junction Temperature and Bondwire Lift-off in Condition Monitoring for
Multichip IGBT Modules," CIPS 2018; 10th International Conference on Integrated Power Electronics Systems, Stuttgart, Germany, 2018, pp. 1-6.

[44] R. Simpson, A. Plumpton, M. Varley, C. Tonner, P. Taylor and X. Dai, "Press-pack IGBTs for HVDC and FACTs," in CSEE Journal of Power and Energy Systems, vol. 3, no. 3, pp. 302-310, Sept. 2017. 